\title{
TERAPIA FOTODINÁMICA EN CORIORRETINOPATÍA SEROSA CENTRAL CRÓNICA SEVERA
}

\section{PHOTODYNAMIC THERAPY IN SEVERE CHRONIC CENTRAL SEROUS CHORIORETINOPATY}

\author{
RECHE-FRUTOS J ${ }^{1}$, CALVO-GONZÁLEZ C ${ }^{1}$, DONATE-LÓPEZ J ${ }^{1}$, \\ SÁENZ-FRANCÉS-SAN-BALDOMERO F ${ }^{1}$, CERVÁN-LÓPEZ I ${ }^{1}$, GARCÍA-FEIJOÓ J², \\ GARCÍA-SÁNCHEZ J²
}

\section{RESUMEN}

Objetivo: Determinar la eficacia y seguridad de la terapia fotodinámica en coriorretinopatía serosa central crónica (CSC)

Métodos: Se incluyen pacientes con formas crónicas de CSC y signos de actividad de la enfermedad tratados con terapia fotodinámica. Se lleva a cabo una exploración oftamológica completa incluyendo la medida de mejor agudeza visual corregida (MAVC) en optotipo ETDRS, angiografía con fluoresceína (AFG) y verde de indocianina (AVI) y tomografía de coherencia óptica (OCT). El objetivo primario del estudio es determinar el cambio medio en agudeza visual.

Resultados: Se han incluido 11 ojos de 11 pacientes. La MAVC media ha pasado de 20/76 a 20/64. El 35\% de los ojos mejoraron su MAVC en dos o más líneas, en el $45 \%$ se mantuvo estable y el $18 \%$ perdieron dos o más líneas. En todos los casos se redujo la hiperpermeabilidad coroidea y en un $80 \%$ el desprendimiento de retina neurosensorial. Todos los pacientes recibieron un solo tratamiento salvo un caso que requirió dos por la aparición de una neovascularización coroidea (NVC). En otro caso

\begin{abstract}
Objective: To determine the efficacy of Photodynamic Therapy (PDT) in chronic Central Serous Chorioretinopathy (CSC).

Methods: Patients diagnosed with chronic CSC, with clinical evidence of activity and treated with Photodynamic Therapy, are included in this report. All were assessed by a complete ophthalmological examination, including assessment of the best corrected visual acuity (BCVA) using an ETDRS chart, fluorescein and indocyanine angiography and optical coherence tomography (OCT). The main objective of the study was to determine the mean visual acuity change.
\end{abstract}

Results: 11 eyes of 11 patients were included in the study, which had a mean follow-up period of 11 months. The mean BCVA increased from 20/76 to $20 / 64.35 \%$ of eyes improved their BCVA by 2 lines or more, $45 \%$ remained stable and $18 \%$ lost 2 lines or more. Choroidal hyperpermeability was reduced in every case. Neurosensorial retinal detachment decreased in $80 \%$ of cases. Only one eye received a second PDT treatment due to choroidal neovascularization. An increase of atrophy over the Retinal

\footnotetext{
Recibido: 14/12/06. Aceptado: 28/11/07.

Sección de Retina. Servicio de Oftalmología. Hospital Clínico San Carlos. Madrid. España.

1 Licenciado en Medicina.

2 Doctor en Medicina.

Correspondencia:

Juan Reche Frutos

Servicio de Oftalmología

Hospital Clínico San Carlos

Avda. Prof. Martín Lagos, s/n

28040 Madrid

España

E-mail: rechejuan@yahoo.es
} 
se apreció incremento de la atrofia sobre el epitelio pigmentario de la retina (EPR). El seguimiento medio ha sido de 11 meses.

Conclusiones: La administración de TFD en pacientes con CSC crónica mejora especialmente los signos de actividad de la enfermedad, como la hiperpermeabilidad coroidea o el desprendimiento de retina neurosensorial. La mejoría en la agudeza visual, sin embargo, no es constante, probablemente debido a la alteración en el EPR.

Palabras clave: Terapia fotodinámica, coriorretinopatía serosa central crónica, tomografía de coherencia óptica, angiografía con verde de indocianina, hipermeabilidad coroidea.
Pigment Epithelium (RPE) was observed in another patient.

Conclusions: PDT can reduce the clinical signs of activity, such as choroidal hyperpermeability or neurosensorial retinal detachment, in patients affected by chronic CSC. However, the increase in visual acuity is variable, probably due to the extent of RPE damage (Arch Soc Esp Oftalmol 2008; 83: 9-14).

Key words: Photodynamic therapy, chronic central serous chorioretinopathy, optical coherence tomography, indocyanine green angiography, choroidal hyperpermeability.

\section{INTRODUCCIÓN}

De forma tradicional, se ha considerado a la coriorretinopatía serosa central (CSC) como un trastorno idiopático de la barrera retinocoroidea caracterizado por un desprendimiento seroso de la retina neurosensorial, debido al paso de líquido a través del epitelio pigmentario de la retina (EPR) (1).

En la mayoría de los casos, se considera un trastorno benigno, más frecuente en varones jóvenes, que suele cursar con resolución espontánea del cuadro y recuperación visual. En algunos casos, evoluciona a una forma recurrente, con la aparición de brotes en el tiempo, considerándose entonces una CSC subaguda (2).

Existe, sin embargo un 5\% de casos en los que se produce una pérdida visual severa, debido a la afectación progresiva del EPR. Esta forma de enfermedad, llamada CSC crónica, suele afectar a pacientes más mayores. Se caracteriza por presentar en la exploración, áreas de atrofia del EPR parcheadas, con cambios en la distribución de pigmento y zonas de desprendimiento del epitelio pigmentario de la retina. A pesar de su carácter crónico, cursa con exacerbaciones y remisiones, siendo su pronóstico visual final pobre (3).

Diversos autores han utilizado la terapia fotodinámica (TFD) en el tratamiento de la CSC subaguda y crónica, basándose en la reducción de la permeabilidad coroidea observada tras la administración de dicho tratamiento (4). En la actualidad, los resultados obtenidos difieren de forma significativa.

El objetivo del trabajo es valorar el cambio en la AV de pacientes con formas crónicas y severas de
CSC activas tratadas con TFD. Como objetivos secundarios se pretende evaluar el número de retratamientos necesarios con TFD durante un período medio de seguimiento aproximado de un año, así como los cambios angiográficos y tomográficos observados tras la TFD.

\section{SUJETOS, MATERIAL Y MÉTODOS}

Se han incluido once ojos de once pacientes con CSC crónica, con signos de actividad reciente.

Los criterios de inclusión han sido: pacientes con CSC crónica, entendiendo como tal la presencia de una daño significativo en el EPR, especialmente a nivel subfoveal (evidenciado por retinografía o AFG), en los que, además existían signos de exacerbación o actividad del proceso, tales como hiperpermeabilidad coroidea en angiografía con verde de indocianina (AVI) o presencia de desprendimiento de retina neurosensorial o de EPR en la tomografía de coherencia óptica (OCT) (fig. 1).

Se han excluido del estudio, formas subagudas en los que el EPR no estaba significativamente afectado y formas crónicas que no presentaban signos de actividad.

Asimismo, se han excluido los pacientes en los que la administración de TFD estaba contraindicada, así como aquellos casos susceptibles en la exploración de presentar una neovascularización coroidea (NVC).

La exploración oftalmológica incluía: determinación de mejor agudeza visual corregida (MAVC) según optotipo ETDRS, retinografía, angiografía con fluoresceína y verde de indocianina y tomogra- 


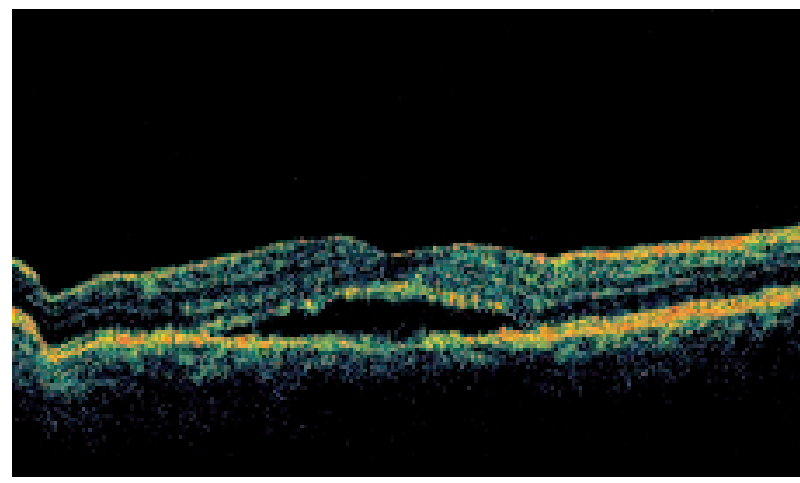

Fig. 1: Desprendimiento de retina neurosensorial y alteración en el segmento externo de los fotorreceptores en la OCT foveolar.

fía de coherencia óptica. Dicha exploración fue realizada antes y después del tratamiento con TFD y cada tres meses.

La administración de TFD ha sido realizada según los parámetros habituales con una infusión de verteporfina (Visudyne, Novartis, Basilea, Suiza). El tamaño del spot se ha determinado según el área de aumento de permeabilidad coroidea encontrada en la AVI.

En todos los casos se realizó una sesión de TFD al inicio del estudio y posteriores revisiones cada tres meses valorando en cada una de ellas la necesidad de retratamiento.

\section{RESULTADOS}

Se han incluido en el estudio once ojos de once pacientes, siete hombres y cuatro mujeres. La edad media fue 53,2 años (rango, 42 a 63 años).

La agudeza visual inicial media era de 20/76 y la final de 20/64. El 36,3\% (4/11 ojos) mejoraron dos o más líneas su agudeza visual, el 45,5\% (cinco ojos) se mantuvieron estables y el 18,2\% (dos ojos) perdieron dos o más líneas de agudeza visual.

Todos los pacientes presentaban un daño severo del EPR a nivel subfoveal, así como una fuga tardía de contraste, evidenciado en la AFG. En tres casos $(27,3 \%)$ se observó, además, un punto de fuga localizado. Tras la administración de TFD, dicho punto de fuga localizado se atenuó en los tres casos (figs. 2 y 3). No hubo mejoría de la alteración del EPR ni de la fuga tardía de contraste.

Las placas de hiperpermeabilidad coroidea evidenciadas en tiempos medios de la AVI presentes en

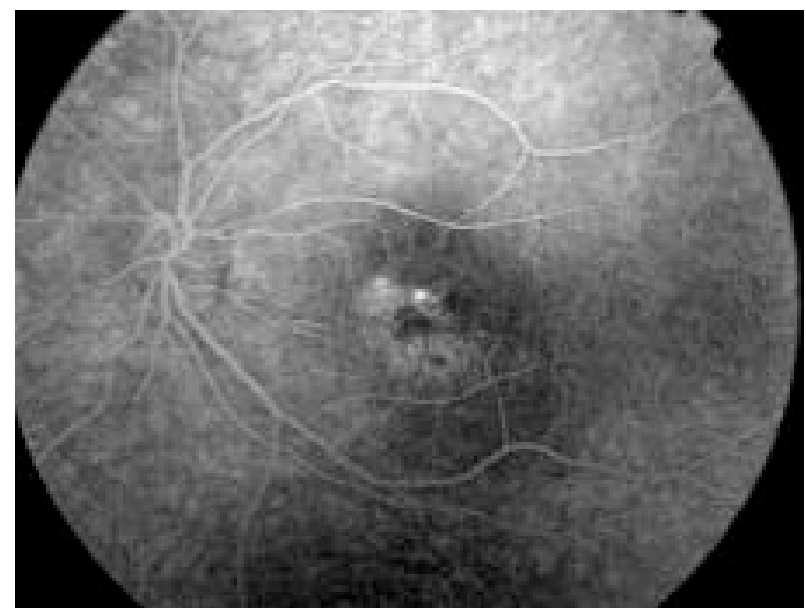

Fig. 2: Punto de fuga en la $A F G$.

todos los ojos mejoraron tras la administración de TFD en el $100 \%$ de los ojos (figs. 4 y 5).

Respecto a los hallazgos encontrados en la OCT, todos los ojos del estudio presentaban un área de desprendimiento de retina neurosensorial. En el $63,6 \%$ era evidente la irregularidad del segmento externo de la capa de fotorreceptores, en el 27,3\% presentaban áreas de desprendimiento de EPR asociadas y en $36,4 \%$ existían zonas hiperreflectantes (depósitos de fibrina) dentro del desprendimiento de retina neurosensorial.

Tras la administración de TFD, se apreció una resolución del desprendimiento de retina neurosensorial en nueve de los once pacientes (80\%). Sin embargo, la irregularidad en capa de fotorrecepto-

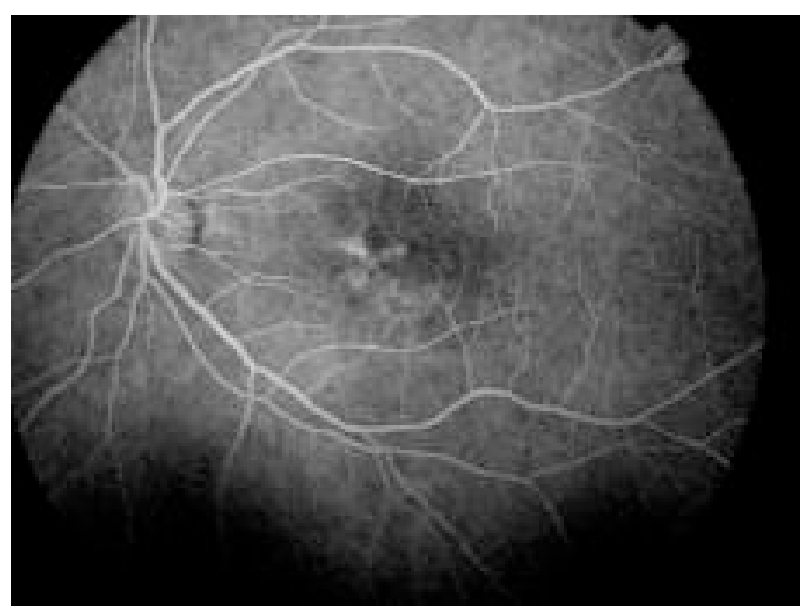

Fig. 3: Atenuación del punto de fuga en la AFG tras TFD. 


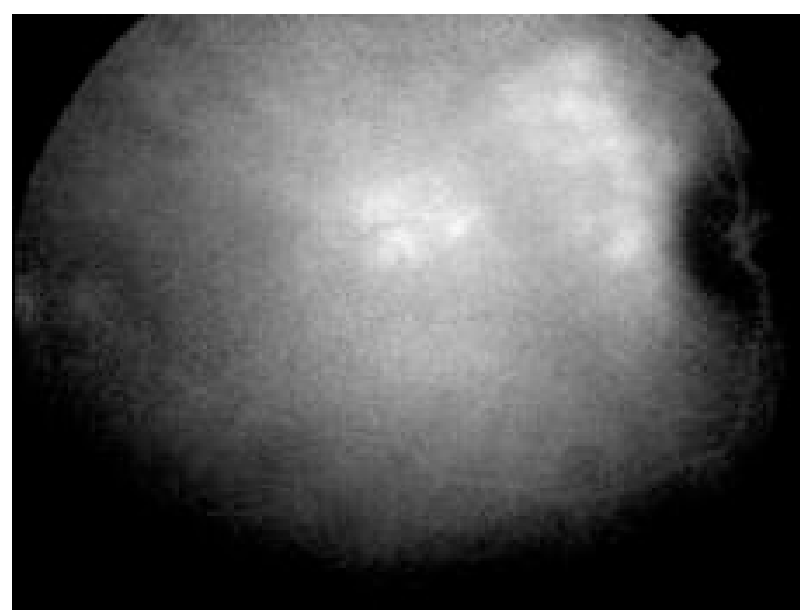

Fig. 4: Placas de hiperpermeabilidad coroidea en la AVI.

res o el desprendimiento de EPR no mostraron cambios significativos (fig. 5).

Un paciente $(9,1 \%)$, presentó a los tres meses de la TFD, una NVC clásica de pequeño tamaño, por la que precisó una segunda sesión de TFD, con el spot localizado exclusivamente en el área de NVC.

Ningún otro paciente recibió una segunda sesión de terapia fotodinámica.

El tiempo de seguimiento medio fue de 11 meses (rango de 8 a 19).

\section{DISCUSIÓN}

La CSC tiene, en la mayoría de los casos, un curso benigno con escasa repercusión sobre la agudeza

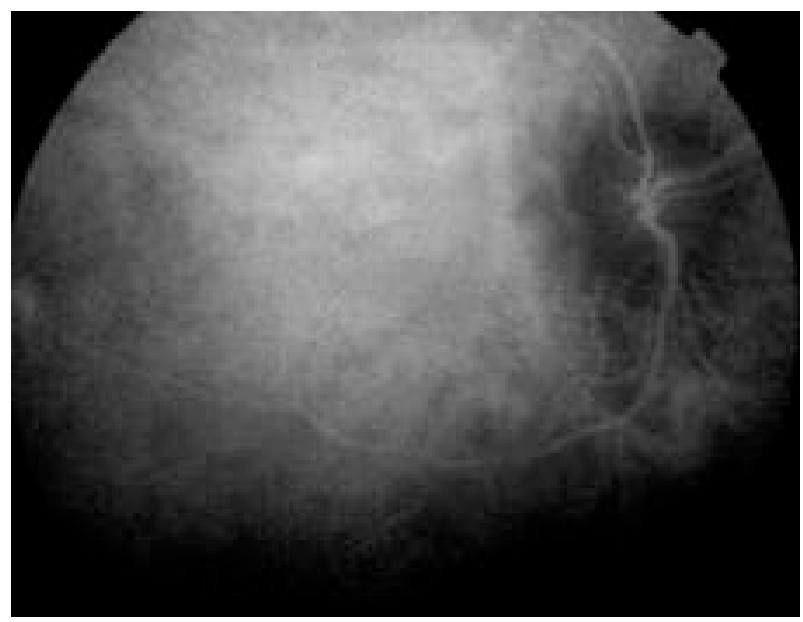

Fig. 5: Atenuación de la hiperpermeabilidad coroidea en la AVI. visual final y un buen pronóstico sin necesidad de tratamiento. Ocasionalmente, los brotes de actividad de la enfermedad pueden tener un curso recurrente o bien no resolverse espontáneamente en pocos meses; son formas subagudas recurrentes o persistentes.

Existen, sin embargo un grupo de pacientes, generalmente de mayor edad y con una evolución de la enfermedad más prolongada, en los que el pronóstico visual es mucho peor debido al daño progresivo producido en el EPR. En este sentido, el concepto de CSC crónica se asocia, más que a un concepto cronológico de tiempo de evolución de la enfermedad, a un daño significativo del EPR.

Varios autores (5-8) han tratado formas subagudas o crónicas de la enfermedad con TFD, obteniendo diversos resultados. No obstante, es necesario mencionar que existe una limitación importante en todos los estudios sobre CSC crónica realizados, ya que los criterios para considerar a una CSC como crónica no están universalmente aceptados ni basados en hallazgos objetivos. En nuestro estudio, se han incluido pacientes con una afectación severa y de localización subfoveal del EPR, basándonos en la imagen retinográfica, de la AFG y del OCT. Adicionalmente, estos pacientes presentaban signos de actividad o exacerbación de la enfermedad como eran la hiperpermeabilidad coroidea o el desprendimiento de retina neurosensorial en el OCT, susceptibles de mejorar con la administración de TFD.

La introducción de la AVI ha puesto de manifiesto la existencia de placas de hiperpermeabilidad coroidea con fuga de líquido al espacio extravascular en pacientes con CSC. Este hallazgo ha cambiado, en parte, la teoría tradicional de que el origen de la CSC se sitúa en un punto localizado de disfunción del EPR, señalizado por la AFG.

En este sentido, existe cierta controversia acerca del tamaño del spot y la prueba más adecuada para guiar el tratamiento con TFD.

Algunos autores, tratan con spots pequeños basándose en el punto de fuga de la AFG. Según esta teoría, no es necesario tratar según la AVI, ya que la hiperpermeabilidad coroidea también puede aparecer en zonas donde el aspecto retinográfico y angiográfico es aparentemente normal (5).

Otros trabajos guían la TFD por el hallazgo de la AVI, con la razón de que con ello se trata el proceso a nivel fisiopatológico, entendiendo que el origen de la enfermedad está en la hiperpermeabilidad coroidea y no en la disfunción localizada del EPR (7). 
En nuestro caso, se ha seleccionado el tamaño del spot de TFD basándonos en la placa de hiperpermeabilidad coroidea hallada en la AVI, ya que dicho hallazgo era constante en todos los ojos afectados, mientras que la existencia de puntos de fuga en AFG era muy infrecuente.

En cuanto a los resultados visuales, la agudeza visual media no experimenta cambios significativos en la serie. El porcentaje de pacientes que mejora es de un $35 \%$. En otras series, este porcentaje es muy variable, destacando un $30 \%$ en la serie de Yanuzzi (7) (6/20 ojos) y el $100 \%$ de la serie de Taban (5) (5/5 ojos). Como ya se ha comentado, los criterios de inclusión de estos pacientes varían de un estudio a otro, y, con ello, la gravedad de la enfermedad. Este hecho ha llevado a sugerir que la mejoría visual es más esperable en aquellos pacientes con daño menos severo del EPR.

En relación a los hallazgos de la pruebas de imagen, existe un mayor consenso en la literatura. Nuestra serie documenta una resolución del desprendimiento de retina neurosensorial en el OCT en el $80 \%$ de ojos, cifra más próxima al $60 \%$ de Yannuzzi (7) o al 100\% de Taban (5).

Asimismo, la reducción de la permeabilidad coroidea en todos los casos de nuestra serie es un hallazgo compartido por estos autores. En la serie de Chan (6), se objetiva una reducción en el calibre de los vasos coroideos dilatados de 546 a 371 micras, con reducción en la fuga al espacio extravascular.

Dos casos de nuestra serie perdieron dos líneas de visión. En uno de los casos, se constató el desarrollo de una NVC tras la administración de TFD. Se ha descrito la aparición de NVC en un 4 a $8 \%$ de los casos de CSC (2), como consecuencia de la disrupción del EPR. Asimismo, se ha descrito un caso similar en la serie de Chan (6), en el que se especula que la NVC también puede aparecer como consecuencia de la isquemia localizada en la coriocapilar inducida por la propia TFD. En este último estudio, la NVC se resolvió espontáneamente. En el nuestro, se estabilizó tras una segunda sesión de TFD, únicamente localizada en la zona de la NVC.

Un segundo paciente perdió agudeza visual a pesar de mejorar el aspecto en AVI y OCT. Es este caso, sin embargo, se constató un incremento significativo en la atrofia del EPR en la retinografía y AFG tras la administración de TFD. Dicho efecto nocivo de la TFD sobre el EPR ha sido ya descrito (9). Debido a este motivo, algunos autores han pro- puesto la reducción de la dosis de TFD para reducir la toxicidad sobre el EPR (10).

En conclusión, podemos afirmar que la administración de TFD en pacientes con CSC crónica mejora especialmente los signos de actividad de la enfermedad, como la hiperpermeabilidad coroidea o el desprendimiento de retina neurosensorial. La mejoría en la agudeza visual, sin embargo, parece más dependiente del estado del EPR.

La presencia de potenciales complicaciones hace necesario tomar ciertas precauciones a la hora de administrar este tratamiento.

Son necesarios más estudios, con períodos de seguimientos más amplios para contrastar estos resultados y resolver algunas de las cuestiones aquí planteadas.

\section{BIBLIOGRAFÍA}

1. Ciardella AP, Guyer M, Spitznas M, Yannuzzi LA. Central serous chorioretinopathy. In: Ryan SJ. Retina. 3rd Edition; St Louis: Mosby; 2001; 1153-1181.

2. Spaide RF, Campeas L, Haas A, Yanuzzi LA, Fisher YL, Guyer DR, et al. Central serous chorioretinopathy in younger and older adults. Ophthalmology 1996; 103: 2070-2079.

3. Schatz H, Madeira D, Johnson RN, McDonald HR. Central serous chorioretinopathy in patients 60 years of age and older. Ophthalmology 1992; 99: 63-67.

4. Schmidt-Erfurth U, Michels S, Barbazetto I, Laqua $H$. Photodynamic effects on choroidal neovascularization and physiological choroid. Invest Ophthalmol Vis Sci 2002; 43: 830-841.

5. Taban M, Boyer DS, Thomas EL, Taban M. Chronic central serous chorioretinopathy: photodynamic therapy. Am J Ophthalmol 2004; 137: 1073-1080.

6. Chan WM, Lam DS, Lai TY, Tam BS, Liu DT, Chan CK. Choroidal vascular remodelling in central serous chorioretinopathy after indocyanine green guided photodynamic therapy with verteporfin: a novel treatment at the primary disease level. Br J Ophthalmol 2003; 87: 1453-1458.

7. Yannuzzi LA, Slakter JS, Gross NE, Spaide RF, Costa DL, Huang SJ, et al. Indocyanine green angiography-guided photodynamic therapy for treatment of chronic central serous chorioretinopathy: a pilot study. Retina 2003; 23 . 288-298.

8. Battaglia Parodi M, Da Pozzo S, Ravalico G. Photodynamic therapy in chronic central serous chorioretinopathy. Retina 2003; 23: 235-237.

9. Lai TY, Chan WM, Lam DS. Transient reduction in retinal function revealed by multifocal electroretinogram after photodynamic therapy. Am J Ophthalmol 2004; 137: 826833.

10. Lai TY, Chan WM, Li H, Lai RY, Liu DT, Lam DS. Safety enhanced photodynamic therapy with half dose verteporfin for chronic central serous chorioretinopathy: a short term pilot study. Br J Ophthalmol 2006; 90: 869-874. 